\title{
LA TRADUCCIÓN DE LOS CUESTIONARIOS DE SALUD PARA PACIENTES
}

\author{
The translation of Care Questionnaires for Patients
}

\author{
Lucila María Pérez Fernández.
}

Patricia Miaja Menéndez,*

\begin{abstract}
RESUMEN
Los cuestionarios de salud y calidad de vida para pacientes son una valiosa herramienta para evaluar la salud, tanto a nivel individual como poblacional. Tal es la importancia que esta herramienta está adquiriendo, que su traducción y buena adaptación también se hacen relevantes. El objetivo de este artículo es determinar la validez lingüística y cultural de diversos cuestionarios. Por ello, se ha llevado a cabo una comparación de las versiones originales y las traducciones validadas en español de cinco cuestionarios que evalúan trastornos de dependencia al alcohol, de adicción al juego, los síntomas de la ansiedad, las conductas alimentarias y los síntomas de la depresión. Dicho análisis se ha efectuado de acuerdo con diversos parámetros lingüísticos, socioculturales y visuales. Los resultados obtenidos nos han permitido desarrollar una serie de indicaciones que se deben tener en cuenta a la hora de traducir cuestionarios de salud.
\end{abstract}

Palabras clave: Cuestionarios de salud y calidad de vida, análisis comparativo, cuestionario, traducción, equivalencia.

\begin{abstract}
Care Questionnaires for patients are an important tool to assess health, both at the population and individual level. Such is the importance that this tool is acquiring, that its translation and good adaptation also become relevant. The objective of this article is to determine the linguistic and cultural validity of various questionnaires. Therefore, we have compared the original versions and the validated Spanish translations of five questionnaires that assess alcohol dependence disorders, gambling addiction, anxiety symptoms, eating behaviors and depression symptoms. This analysis has been carried out according to various linguistic, sociocultural and visual parameters. The results obtained have allowed us to develop some indications that must be taken into account when translating health questionnaires.
\end{abstract}

Key Words: Care Questionnaires for patients, comparison, questionnaire, translation, equivalence.

*Universidad de Málaga. Investigadora. España. Correo electrónico: perezfernandezlucila@gmail.com ** Universidad Europea del Atlántico. Traductora. España. Correo electrónico: patriciamiaja@gmail.com 


\section{Introducción}

Los cuestionarios de salud y calidad de vida son instrumentos creados por la comunidad científica para obtener información sobre el estado de salud de los ciudadanos de un área geográfica determinada. También sirven para que los propios pacientes sepan más acerca de su calidad de vida y su salud. El desarrollo de estos documentos como instrumentos de medición de la salud y la calidad de vida se hace necesario si se quiere que tengan aplicabilidad clínica. Para ello, se necesita comprobar que los cuestionarios son fiables, es decir, muestran la realidad de cómo se siente el paciente, con el objetivo de ofrecer el diagnóstico más adecuado en función de la gravedad e índice de afección de la enfermedad que se evalúa. Por medio de estos cuestionarios se mejora la atención sanitaria y se determina el impacto de una enfermedad, afección o trastorno, así como su gravedad.

Dado que su objetivo es ofrecer un diagnóstico y mejorar la calidad de vida de los pacientes, la traducción y la adaptación de estos documentos también es importante, debido a que la falta de comprensión, sea por cuestiones lingüísticas o culturales, podría afectar los resultados del cuestionario y, por lo tanto, el diagnóstico no se adecuaría a las necesidades del usuario. También afectaría a la comunidad científica, puesto que la información que obtienen sobre la afección de las enfermedades no es la correcta.

El objetivo del presente artículo es determinar la validez tanto lingüística como cultural de cinco cuestionarios. Para ello, se plantean dos hipótesis principales. En primer lugar, dado que se trata de instrumentos dirigidos a pacientes legos en medicina, se espera no encontrar ningún elemento que dificulte su lectura y comprensión. Por ende, no contendrán terminología especializada. En segundo lugar, si se considera que los pacientes deberán rellenar dichos cuestionarios, se supone que la traducción de los cuestionarios se ajusta a las necesidades del público en cuanto a formato, tipografía, 
imágenes, de tal forma que estos factores no supongan una dificultad a la hora de responderlos y evitar que repercutan en los resultados.

\section{Los cuestionarios de salud y calidad de vida}

Congost (2016) define los cuestionarios de salud y calidad de vida como "instrumentos dirigidos a un público amplio donde se presentan, de forma clara, neutra y objetiva, determinados aspectos relacionados con la enfermedad y el tratamiento, es decir, con el estado funcional y el bienestar emocional del paciente" (p. 117). Estos aportan información sobre la salud, a nivel individual y colectivo, a pacientes que padecen o no una enfermedad; existen dos tipos de cuestionarios: generales y específicos. Los cuestionarios generales ofrecen información sobre el estado global de salud de los pacientes. Los específicos proporcionan información detallada sobre una enfermedad determinada. Los cuestionarios de salud y calidad de vida despiertan un interés en la comunidad científica cada vez mayor. Como consecuencia de la disminución de la mortalidad y el aumento de la natalidad que se ha experimentado en el último siglo, las enfermedades crónicas o degenerativas e incapacidades cada vez son más comunes y conocidas (Congost, 2010), por lo que estos cuestionarios son la forma en la que los médicos y el personal sanitario obtienen información sobre el estado de la población de un área geográfica definida (una ciudad, una región, un país, etc.), al mismo tiempo que el individuo conoce su estado de salud.

\subsection{La traducción y la adaptación de los cuestionarios de salud y calidad de vida}

El objetivo de los cuestionarios de salud y calidad de vida es aportar información, tanto a la comunidad científica sobre la salud de la población de una zona geográfica determinada como al paciente sobre su salud y la enfermedad de la que trata el propio 
cuestionario. A partir de la información que aporta a la comunidad científica, estos cuestionarios han ganado importancia como unidad métrica e informativa. Esta relevancia es la que ha generado interés en los profesionales de la lengua por su traducción y adaptación, centrándose en la equivalencia (tanto lingüística como cultural) entre las versiones de los cuestionarios, que determina si la funcionalidad de estos es la misma.

Los miembros de la comunidad científica se intercambian datos a nivel internacional, por lo que, según Congost (2010) "es preferible utilizar cuestionarios adaptados para llevar a cabo estudios comparativos interculturales” (p. 46). Por lo tanto, esta autora afirma que la traducción y la adaptación de estos textos son relevantes porque se estandarizan las medidas para la comunidad, permiten compartir y comparar los datos con el resto de los países, y ahorran tiempo y dinero en la creación de otros cuestionarios.

Congost (2018) señala que el hecho de que los cuestionarios se hayan creado en países como Estados Unidos y Gran Bretaña “permite la homologación de nuestras investigaciones en el ámbito internacional, la participación en proyectos multicéntricos y el desarrollo de análisis comparativos de tipo intercultural" (p. 134). Esta autora también resalta el incremento de la demanda de este tipo de instrumentos, lo que hace que se obtengan "datos procedentes de muestras más amplias y con una mayor diversidad cultural"' (p. 134).

Congost (2010) expone las razones por las cuales cree necesarias la traducción y la adaptación de este tipo de documentos. La primera se relaciona con el ahorro de tiempo, pues para desarrollar un cuestionario normalmente se necesitan entre uno y seis años, mientras que su traducción y adaptación cultural es mucho más rápida (de unos meses a dos años como máximo). Asimismo, al optar por la traducción en lugar de la creación 
de un nuevo instrumento, el precio del proceso se reduce en gran medida. La segunda razón tiene que ver con el aprovechamiento de la experiencia de los autores originales de este tipo de instrumentos. Según autores como Brislin (1973) y Hambleton (1996), citados en Congost (2010), hay países que no pueden crear instrumentos validados porque no cuentan con la experiencia técnica para llevar a cabo estos proyectos. De esta forma, los investigadores pueden sentirse más seguros al utilizar una versión adaptada que usando uno nuevo. La tercera y última razón es porque permite comparar los datos obtenidos a nivel nacional e internacional.

En la cultura no solo influyen los aspectos lingüísticos, sino también otros como las costumbres de una comunidad, basadas en una tradición histórica, que es determinante a la hora de responder los cuestionarios, aspectos que deben tomarse en cuenta a la hora de traducirlos y adaptarlos. Por ello, el traductor debe tener conocimiento de ambas lenguas y culturas, con el objetivo de realizar una buena adaptación, no solo de los textos, sino de los contextos en los cuales estos se enmarcan, así como el aspecto pragmático que influye en ellos, pues podría haber aspectos culturales que afecten la comprensión de los cuestionarios. Al respecto, Congost (2010) indica lo siguiente:

El traductor debe poseer un saber pragmático exhaustivo de la cultura de las comunidades lingüísticas a las que sirve de puente, para salvar cualquier diferencia que pueda surgir en la adaptación de los cuestionarios. La falta de simetría cultural o anisomorfismo parte de la idea de que cada lengua tiene su propio genio y personalidad, lo cual implica una diferencia cultural derivada de la historia, las instituciones y los hábitos que les son propios a cada comunidad y que son conceptos específicos de una cultura determinada (p. 16). 
Por otro lado, Martín (2004) añade:

El proceso de traducción y adaptación de una escala requiere algo más que la traducción de la lengua origen a la lengua de destino: es necesario asegurar que las puntuaciones obtenidas con el test traducido son equivalentes a las obtenidas con el test original. Para alcanzar esa equivalencia, hay que considerar cuatro aspectos del proceso: 1) el contexto cultural donde se va a realizar la adaptación, 2) aspectos técnicos del propio desarrollo y adaptación del test, 3) administración del test, y 4) interpretación de las puntuaciones (p. 28).

Así pues, Martín insiste en que el cuestionario tenga la misma funcionalidad pragmática para que no existan grandes diferencias en los resultados entre las dos culturas. De este modo, las propiedades métricas son lo más importante que se deben conservar en la traducción y adaptación de los cuestionarios de salud y calidad de vida para que la interpretación de los resultados sea la misma; es decir, la equivalencia que prima es la métrica (todos los aspectos que influyan en la puntuación y que repercutan en los resultados). Por consiguiente, la fiabilidad de las traducciones de los cuestionarios se determina en función de los resultados, mediante una comparativa que establezca que las diferencias entre ellos no tengan relación con los procesos de traducción y adaptación.

Una de las características más importantes que deben cumplir los cuestionarios de salud es la de fiabilidad, la cual, según Carvajal et al. (2011), se define como "la propiedad que designa la constancia y precisión de los resultados que obtienen un instrumento al aplicarlo en distintas ocasiones" (p. 66). Añaden, además, que "evalúa el grado de consistencia en que un instrumento mide lo que debe medir [y que] un instrumento es fiable cuando los resultados son comparables en situaciones similares" (p. 66). Martín (2004) indica al respecto que "la fiabilidad de un instrumento de medida 
se valora a través de la consistencia, la estabilidad temporal y la concordancia interobservadores" (p. 27). La consistencia evalúa la relación entre los ítems de la escala; la estabilidad temporal es la validez que tiene la escala en distintas situaciones temporales, valorada por el mismo profesional; la concordancia interobservadores analiza la equivalencia entre los resultados al ser evaluada por dos profesionales diferentes o en distintas situaciones temporales.

Por su parte, Carvajal et al. (2011) hablan del concepto de la validez, que definen como "una pieza clave tanto en el diseño de un cuestionario como en la comprobación de la utilidad de la medida realizada" (p. 69). Estos autores, al igual que Martín (2004), distinguen tres tipos de validez: de contenido, de constructo y de criterio. La primera evalúa si los ítems del cuestionario "son indicadores de lo que se pretende medir" (Martín, 2004, p. 5). La segunda, según esta última autora, "evalúa el grado en que el instrumento refleja la teoría del fenómeno o del concepto que mide” (p. 6). Por último, la tercera evalúa la "relación de la puntuación de cada sujeto con un Gold Standard que tenga garantías de medir lo que deseamos medir” (Martín, 2004, p. 6).

En cuanto al proceso de traducción al cual se someten los cuestionarios, el modelo de retrotraducción es el método más habitual y se desarrolla en diferentes fases. Si se parte de la combinación lingüística inglés-español, el proceso es el siguiente. En primer lugar, un grupo de traductores españoles trabajan individualmente y hacen, al menos, dos versiones traducidas a la lengua meta del cuestionario original. A continuación, estos traductores, junto con los autores originales, ponen en común los documentos para realizar una primera versión. En segundo lugar, otro grupo de traductores anglosajones, que también trabajan de forma individual, traducen esta versión al inglés sin haber visto el original. Junto con los autores, vuelven a realizar una segunda versión del documento, proceso que se repite hasta que las versiones en inglés 
(la original y la retrotraducida) sean iguales o, por lo menos, no haya diferencias significativas entre ellas. En tercer y último lugar, se reúne a un grupo de personas (de características similares a la población meta) para realizar una primera prueba de la versión traducida, con el fin de comprobar que no hay problemas de comprensión. De acuerdo con Congost (2018):

Esta prueba es una de las más importantes en lo que se ha decidido llamar "control de calidad" de la traducción final, donde la versión final del cuestionario se somete a una prueba preliminar o estudio piloto para evaluar su equivalencia con el texto original (p. 315).

\subsubsection{Características lingüísticas y problemas traductológicos de los cuestionarios de} salud y calidad de vida

Estos cuestionarios "se identifican con un estilo llano y claro, y su lenguaje se caracteriza, normalmente, por hacer uso de una sintaxis sencilla, utilizar frases cortas, usar la voz activa y evitar tiempos verbales complicados" (Congost, 2016, p. 117). Sin embargo, aunque en ellos se prime un lenguaje poco especializado, estos textos poseen características lingüísticas propias que pueden dar problemas a la hora de traducirlos. Entre las más frecuentes, Congost (2016) señala las siguientes: polisemia, secuenciación terminológica, repetición léxica, registro y variación léxica, y estilo.

a) Polisemia

Las palabras polisémicas son características del lenguaje común, aunque también las encontramos en el lenguaje especializado. Son palabras que tienen más de un significado, por lo que su traducción es uno de los obstáculos a los que tiene que enfrentarse el traductor de textos científicos. 
b) Secuenciación terminológica

Cada lengua presenta un orden o secuenciación de aparición de ciertos términos, por ejemplo, on-off, true-false o yes-no. Si alteramos el orden natural en la lengua meta, es probable que el lector se muestre confundido y esto repercuta en las respuestas seleccionadas.

c) Repetición léxica

En determinadas lenguas las repeticiones léxicas encajan mejor, pero en otras, como en español, no. Por lo tanto, es necesario tener en cuenta este aspecto con el fin de no producir textos repetitivos en español.

d) Registro y variación léxica

Los textos que se dirigen a un público general se caracterizan por la presencia de un registro medio, en el que se prescinda de terminología especializada. Asimismo, en el caso del español se intenta optar por la variedad del español neutro, con el fin de evitar regionalismos que puedan entorpecer la comprensión.

e) Estilo

Se ha visto que el lenguaje empleado en este tipo de textos se caracteriza por su simpleza y concisión, por lo que las oraciones que aparecen son cortas y simples. Congost (2016) lo explica muy bien cuando dice que "los cuestionarios de salud se identifican con un estilo llano y claro, y su lenguaje se caracteriza, normalmente, por hacer uso de una sintaxis sencilla, utilizar frases cortas, usar la voz activa y evitar tiempos verbales complicados" (p. 117). Por lo tanto, lo que se persigue con esto es que el lector entienda lo que aparece en los cuestionarios: si las oraciones son más largas, los lectores podrían perderse y no tener claro el sentido del texto. 


\section{Metodología}

El presente estudio se basa en un análisis comparativo de las traducciones de los cuestionarios de salud y calidad de vida del inglés al español, centrado en tres aspectos: lingüísticos, socioculturales y visuales.

Para ello, se han seleccionado cinco cuestionarios diferentes y sus correspondientes traducciones. Estos abordan trastornos presentes en la sociedad actual, por lo que se cree que su análisis puede resultar de interés para la comunidad científica. A continuación, se presentan los cuestionarios objeto de análisis:

Alcohol Use Disorders Identification Test (AUDIT) > Test AUDIT (validación del cuestionario en España por la OMS para detectar los consumos de riesgo como dependencia alcohólica);

South Oaks Gambling Screen (SOGS) > Cuestionario de juego South Oaks (SOGS);

Anxiety Hamilton Rating Scale > Escala de Ansiedad de Hamilton;

Eating Attitudes Test (EAT-26) > Cuestionario de Actitudes ante la Alimentación (EAT-26);

Beck's Depression Inventory (BDI-2) > Inventario de Depresión de Beck (BDI2).

Como se observa, son cuestionarios específicos, ya que proporcionan información detallada sobre una enfermedad determinada. Por lo tanto, el análisis se basará en las características inherentes a este tipo de cuestionarios y se seguirá la siguiente estructura propuesta por Congost (2010): 
1) Cuestiones lingüísticas

a) Nivel oracional

i) Planos léxico, semántico, y morfosintáctico (sustitución, inclusión, omisión, falsos amigos, expresiones coloquiales, repetición léxica, redundancia léxica y sintáctica, grado de formalidad, polisemia, oraciones largas, complejas o equívocas, tiempos verbales, anglicismos, adverbios acabados en -mente, gerundios, etc.).

ii) Plano ortográfico

b) Nivel supraoracional (todo lo relacionado con el contenido del cuestionario)

2) Cuestiones socioculturales (equivalencias culturales y pragmática)

3) Cuestiones visuales (disposición visual y variables tipográficas)

\section{Análisis de los cuestionarios}

\subsection{Cuestiones lingüísticas}

En este apartado se analizan los niveles oracional y supraoracional de los cuestionarios en estudio.

\subsubsection{Nivel oracional}

El análisis de este nivel se ha efectuado atendiendo a tres planos (morfosintáctico, ortográfico y léxico). A continuación, se exponen los ejemplos más relevantes:

a) Grado de formalidad

Se encuentran diferencias en los cuestionarios en cuanto a la forma de tratamiento de la persona (ver tabla 1). Por ejemplo, el test AUDIT, redactado en 
español, se dirige al paciente de "tú", mientras que el cuestionario SOGS opta por la forma "usted". También en la Escala de Ansiedad de Hamilton y en el BDI-2, al enumerar síntomas, sentimientos y comportamientos, se observa la forma de tratamiento de "usted" en las instrucciones.

Tabla 1. Ejemplos de diferencias en el tratamiento de la persona

\begin{tabular}{ll}
\hline Cuestionario & Texto meta \\
\hline AUDIT & $\begin{array}{l}\text { ¿Con qué frecuencia, en el curso del último año, has } \\
\text { necesitado beber en ayunas para recuperarte después de } \\
\text { haber bebido mucho el día anterior? }\end{array}$ \\
\hline SOGS & $\begin{array}{l}\text { ¿Ha afirmado usted alguna vez haber ganado dinero en el } \\
\text { juego cuando en realidad había perdido? }\end{array}$ \\
\hline Escala de ansiedad de & $\begin{array}{l}\text { Indique la intensidad con que se cumplieron o no, durante } \\
\text { el último mes, los síntomas que se describen en cada uno } \\
\text { de los 14 ítems: }\end{array}$ \\
\hline BDI-2 & $\begin{array}{l}\text { Por favor, lea con atención cada uno de ellos } \\
\text { cuidadosamente. Luego elija (...). Marque (...). } .\end{array}$ \\
\hline
\end{tabular}

Fuente: elaboración propia.

En este mismo apartado, se analiza el registro terminológico. Estos documentos no deben tener una carga terminológica alta, pues están dirigidos a personas legas en medicina, por lo que su vocabulario debe ser coloquial y fácil de comprender. Por lo general, los cuestionarios que se han analizado están bien adaptados en ese sentido; sin embargo, la Escala de Ansiedad de Hamilton presenta inconsistencia en cuanto al registro terminológico, pues se observa la presencia de términos informales y, en otras ocasiones, especializados. A continuación, en la tabla 2 se presentan algunos ejemplos al respecto.

Tabla 2. Ejemplo de registro terminológico en el cuestionario de la Ansiedad de Hamilton

\begin{tabular}{lll}
\hline & Texto original & Texto meta \\
\hline $\begin{array}{l}\text { Ítem 8 (ejemplo de } \\
\text { lenguaje coloquial) }\end{array}$ & tinnitus & zumbido de oídos \\
\hline $\begin{array}{l}\text { Ítem 9 (ejemplo de } \\
\text { lenguaje especializado) }\end{array}$ & missing beating & $\begin{array}{l}\text { extrasístoles (arritmias } \\
\text { cardíacas benignas) }\end{array}$ \\
\hline
\end{tabular}

Fuente: elaboración propia. 
El registro que se emplea en los demás formularios es coloquial, no aparece terminología especializada y se mantiene a lo largo de los textos.

b) Omisiones

Las diferencias culturales entre las sociedades de la lengua origen y la lengua meta pueden provocar que los traductores opten por omitir cuestiones desmesuradamente específicas de una cultura o irrelevantes para una de ellas.

Tabla 3. Ejemplo de diferencias culturales en el cuestionario SOGS

\begin{tabular}{lll}
\hline Cuestionario & Texto original & Texto meta \\
\hline SOGS & $\begin{array}{l}\text { b. bet on horses, dogs } \text { or } \\
\text { other animals (in off-track } \\
\text { betting, at the track } \text { or with } \\
\text { a bookie) }\end{array}$ & $\begin{array}{l}\text { b) apostar en las carreras de } \\
\text { caballos }\end{array}$ \\
\hline SOGS & $\begin{array}{ll}\text { c. bet on sports (parley } \\
\text { cards, with a bookie, or at } \\
\text { jai alai) }\end{array}$ & $\begin{array}{l}\text { c) apostar en el frotón o en } \\
\text { deptes rurales }\end{array}$ \\
\hline
\end{tabular}

Fuente: elaboración propia.

Como se observa en la tabla 3 , en el texto meta se han efectuado algunas omisiones. Con respecto al ítem b, esto puede deberse a que en España apenas quedan canódromos que organicen carreras de galgos. Además, en España tampoco son comunes las tarjetas parlay (grafía correcta del término que en el cuestionario original aparece de forma errónea). Omitir estas modalidades de juego implica que el texto esté mejor adaptado a la cultura meta y, por lo tanto, sea más comprensible para el usuario. Por su parte, el juego jai alai, de origen vasco, llamado también zesta-punta, o "cesta punta". Este juego se traduce en la versión española como “frontón”. Según el artículo “Cesta punta - el deporte que cautivó América”, de Palacios (3 de junio de 2003), este juego se popularizó en Estados Unidos en los años setenta y ochenta, sobre todo en Florida, donde los partidos se celebraban en los casinos, por lo que se realizaban apuestas. Su popularidad empezó a caer a raíz de la huelga de jugadores en 1988, y 
muchos frontones cerraron sus puertas. No obstante, el deporte se sigue practicando en casinos, donde han habilitado espacios para ello, ya que la ley los obliga a que se practique un deporte para obtener la licencia de máquinas tragaperras e instalaciones destinadas a juegos de azar. A pesar de que es un juego olímpico, esta modalidad de frontón es aún desconocida para muchas personas, por lo que los traductores decidieron emplear el deporte en el cual esta se engloba, para que el usuario lo comprenda.

En la Escala de Ansiedad de Hamilton y en el cuestionario SOGS también se hallan omisiones (ver tabla 4). El primer documento tiene que ver con los síntomas enumerados en el ítem 13. Al comparar la versión original con la traducida, se aprecia que el síntoma raising of hair no aparece en el texto meta. Sin embargo, esta omisión no altera la funcionalidad del ítem, puesto que precisa el significado del síntoma anterior (tensión headache). La Organización Mundial de la Salud (2019) indica que existen varios tipos de cefaleas (tensional, en brotes, de los senos parasanales o por uso excesivo de analgésicos). La de tensión (o tensional) se caracteriza porque el dolor lo provoca, entre otros, una tensión en los músculos del cuero cabelludo, que es lo que se indica en raising of hair.

Tabla 4. Ejemplo de omisiones terminológicas en la Escala de Ansiedad de Hamilton

\begin{tabular}{lll}
\hline Cuestionario & Texto original & Texto meta \\
\hline Escala de Ansiedad de & Autonomic Symptoms: & Síntomas del sistema \\
Hamilton & Dry mouth, flushing, pallor, & nervioso autónomo: Boca \\
& tendency to sweat, & seca. Accesos de \\
& giddiness, tension headache, & enrojecimiento. Palidez. \\
& raising of hair. & Tendencia a la sudoración. \\
& & Vértigos. Cefalea (dolor de \\
& cabeza) de tensión. \\
\hline
\end{tabular}

Fuente: elaboración propia.

Por otro lado, en el cuestionario SOGS, la omisión encontrada es más cultural (tabla 5). En la pregunta 3, se ha omitido información al traducir el cuestionario. En la versión original, se especifica la cantidad de bebidas alcohólicas que el paciente o la 
paciente consume en un día, dependiendo del sexo. Empero, en la versión meta no se recoge este matiz. Esta omisión puede deberse a que esa cantidad ya supera la que el Ministerio de Sanidad, Consumo y Bienestar Social establece como "recomendada" para un consumo diario al realizar un test de consumo en el que, al indicar el sexo de la persona, aparece cuál es el consumo diario recomendado para hombres y mujeres (cuatro y dos unidades, respectivamente); puede ser que esta sea la razón por la que se ha decidido eliminar esta distinción que se hace en el texto original, con la finalidad de ahorrar espacio y no confundir al usuario.

Tabla 5. Ejemplo de omisiones culturales en el cuestionario SOGS

\begin{tabular}{lll}
\hline Cuestionario & Texto original & Texto meta \\
\hline SOGS & How often have you had 6 & ¿Con qué frecuencia te \\
& $\begin{array}{l}\text { or more units if female, or } \\
\text { 8 or more if male, on a } \\
\text { single occasion in the last } \\
\text { year? }\end{array}$ & alcohólicas en un solo día? \\
\hline
\end{tabular}

Fuente: elaboración propia.

c) Inclusiones

En este apartado se analizan las inclusiones observables en las traducciones de los cuestionarios. La primera aparece en la Escala de Ansiedad de Hamilton (ver tabla 6) que, como se mencionó, se encuentra en el ítem 13, donde se analizan los síntomas que afectan el sistema nervioso autónomo. En inglés, se muestra que solo aparece Autonomus Symptoms, mientras que en la versión española se necesita especificar de qué se trata y, por lo tanto, el título que encabeza los síntomas es más largo: “síntomas del sistema nervioso autónomo". Asimismo, para traducir el término headache, se ha incluido un paréntesis aclaratorio "cefalea (dolor de cabeza)", probablemente en aras de aclarar el significado del tecnicismo al público meta. 
Tabla 6. Ejemplo de inclusiones explicativas en la Escala de Ansiedad de Hamilton

\begin{tabular}{lll}
\hline Cuestionario & Texto original & Texto meta \\
\hline Escala de Ansiedad de & Autonomous Symptoms: & Síntomas del sistema \\
Hamilton & $\begin{array}{l}\text { Dry mouth, flushing, } \\
\text { pallor, tendency to sweat, } \\
\text { giddiness, tension }\end{array}$ & nervioso autónomo: Boca \\
& seca. Accesos de \\
& enrojecimiento. Palidez. \\
& & Tendencia a la sudoración. \\
& Vértigos. Cefalea (dolor de \\
& cabeza) de tensión. \\
\hline
\end{tabular}

Fuente: elaboración propia.

En este cuestionario se hallan hasta trece inclusiones terminológicas. Sin embargo, en otros cuestionarios no se encuentran tantas, o ninguna, como en el caso del Test AUDIT. Una que resulta muy interesante se ubica en el ítem 6 de la versión española del cuestionario SOGS (tabla 7). Al contrario que en otros cuestionarios, cuyas inclusiones suelen ser terminológicas, esta es gramatical, puesto que se observan dos formas verbales distintas del verbo "tener", mientras que en la versión original solo hay una. En el texto original, se emplea el present perfect como forma verbal. No obstante, en español se halla el presente y el pretérito perfecto compuesto unidos por la conjunción “o”. Según la Fundación del Español Urgente (Fundéu), el pretérito perfecto compuesto "se refiere sobre todo a una acción pasada cuyos resultados siguen vigentes en el presente, objetiva o subjetivamente, o pueden repetirse" (consulta del 24/05/2010, párrafo 2). Además, indica que puede concretarse de distintas formas, entre las que se encuentra el lapso, que "puede ser el día actual o la vida de una persona" (consulta del 24/05/2010, párrafo 2). Dada esta definición y uso del tiempo compuesto, la forma en presente del verbo "tener" es innecesaria, ya que no aporta significado y podría confundir al usuario, pues hace la lectura más confusa. 
Tabla 7. Ejemplo de inclusiones gramaticales en el cuestionario SOGS

\begin{tabular}{lll}
\hline Cuestionario & Texto original & Texto meta \\
\hline SOGS & $\begin{array}{l}\text { 6. Do you feel you have } \\
\text { ever had a problem with } \\
\text { gambling? }\end{array}$ & $\begin{array}{l}\text { 6. ¿cree usted que tiene o } \\
\text { ha tenido alguna vez } \\
\text { problemas con el juego? }\end{array}$ \\
\hline
\end{tabular}

Fuente: elaboración propia.

d) Gramática

En este apartado se estudia la adaptación gramatical en las versiones españolas de los cuestionarios analizados. Aunque, en general, se trata de un aspecto bastante cuidado, sí se observan algunas inconsistencias, especialmente en el cuestionario BDI-2, como se muestra en la tabla 8.

Tabla 8. Ejemplo de errores gramaticales de estructura en el cuestionario BDI-2

\begin{tabular}{lll}
\hline Cuestionario & Texto original & Texto meta \\
\hline BDI-2 & 17. Irritability & 17. Irritabilidad \\
& $\begin{array}{l}\text { 0. I am not more irritable } \\
\text { than usual. }\end{array}$ & $\begin{array}{l}0 \text { No estoy tan irritable que } \\
\text { lo habitual. }\end{array}$
\end{tabular}

Fuente: elaboración propia.

Como se observa, se produce un calco de la estructura comparativa en inglés que en español debería ser "No estoy más irritable de lo habitual”. Otro aspecto gramatical en los textos es la falta de concordancia entre palabras. Según las normas gramaticales de la Real Academia Española (RAE), la concordancia se define como "la coincidencia obligada de determinados accidentes gramaticales (género, número y persona) entre distintos elementos variables de la oración” (Diccionario Panhispánico de Dudas, 2005, apartado 1). En el cuestionario EAT-26, se encuentra una falta de concordancia nominal, que se da entre el artículo y el sustantivo, entre otros: "las comida" (tabla 9). De acuerdo con el criterio utilizado en este estudio, este error puede haberse producido por no revisar el texto bien o por un descuido al escribir (error tipográfico). 
Tabla 9. Ejemplo de falta de concordancia en el cuestionario EAT-26

\begin{tabular}{|c|c|c|}
\hline Cuestionario & Texto original & Texto meta \\
\hline EAT-26 & & $\begin{array}{l}26 . \text { Tengo ganas de } \\
\text { vomitar después de las } \\
\text { comidas }\end{array}$ \\
\hline
\end{tabular}

Fuente: elaboración propia.

e) Ortografía

En este apartado se analizan las cuestiones que afectan las normas ortográficas y tipográficas de la lengua española. Cabe destacar la presencia de varios errores ortográficos que se extienden en las versiones traducidas de los cuestionarios SOGS y BDI-2. Por ejemplo, en el Cuestionario SOGS, en la pregunta del ítem 6, no se ha escrito la mayúscula inicial (tabla 10). Según las normas ortográficas de la RAE, "si la pregunta o la exclamación constituyen la totalidad del enunciado, y sus signos de cierre equivalen a un punto, la primera palabra de la pregunta o la exclamación se escribe con inicial mayúscula" (Diccionario Panhispánico de Dudas, 2005, apartado 3.4.1).

Tabla 10. Ejemplo de error ortográfico en el cuestionario SOGS

\begin{tabular}{|c|c|c|}
\hline Cuestionario & Texto original & Texto meta \\
\hline SOGS & & $\begin{array}{l}\text { ¿cree usted que tiene o ha } \\
\text { tenido alguna vez } \\
\text { problemas con el juego? }\end{array}$ \\
\hline
\end{tabular}

Fuente: elaboración propia.

En el cuestionario BDI-2, los títulos de cada ítem aparecen en mayúsculas (tabla 11). Esto es un rasgo característico del inglés, mientras que en español solo se escribe en mayúscula la primera letra de la primera palabra. Esto se repite en todos los títulos de los ítems de más de una palabra que aparecen en el documento. 
Tabla 11. Ejemplo de un error ortográfico en un título del cuestionario BDI-2

\begin{tabular}{lll}
\hline Cuestionario & Texto original & Texto meta \\
\hline BDI-2 & 4. Loss of Pleasure & 4. Pérdida de Placer \\
\end{tabular}

Fuente: elaboración propia.

f) Redundancias léxicas y sintácticas

En los cuestionarios analizados, se encuentran ejemplos de redundancias. En la tabla 12, se resalta uno extraído de la Escala de Ansiedad de Hamilton, concretamente en el ítem 12, donde se evalúan los síntomas genitourinarios. En la versión española, los dos últimos síntomas que aparecen descritos son "impotencia” y "ausencia de erección”. Según la descripción que recoge el Diccionario de la Lengua Española (DLE), la impotencia se define como "imposibilidad del varón de realizar el coito" (2020, acepción 2). Por eso, resulta redundante que después aparezca “ausencia de erección”.

Tabla 12. Ejemplo de redundancia terminológica en la Escala de Ansiedad de Hamilton

\begin{tabular}{lll}
\hline Cuestionario & Texto original & Texto meta \\
\hline Escala de Ansiedad de & Genitourinary Symptoms & Síntomas genitourinarios: \\
Hamilton & $\begin{array}{l}\text { Frequency of micturition, } \\
\text { urgency of micturition, }\end{array}$ & Micciones frecuentes. \\
amenorrhea, menorrhagia, & Micción imperiosa. & Amenorrea (falta del \\
development of rigidity, & período menstrual). \\
premature ejaculation, loss & Metrorragia (hemorragia \\
of libido, impotence. & genital). Frigidez. \\
& & Eyaculación precoz. \\
& Impotencia. Ausencia de \\
& erección. \\
\hline
\end{tabular}

Fuente: elaboración propia.

En el ítem 4 del cuestionario SOGS, se miran dos repeticiones léxicas en la misma pregunta, pues se utiliza dos veces el verbo "jugar" y el sustantivo "dinero" (ver tabla 13). Se considera que la primera vez que se emplea este verbo debería haberse traducido por "apostar", ya que una de las acepciones de este verbo es "arriesgar cierta cantidad de dinero en la creencia de que algo, como un juego, una contienda deportiva, etc., tendrá tal o cual resultado" (DLE, 2020, acepción 2). 
Tabla 13. Ejemplo de redundancia léxica en el cuestionario SOGS

\begin{tabular}{lll}
\hline Cuestionario & Texto original & Texto meta \\
\hline SOGS & 4. When you gamble, how & 4. Cuando usted juega \\
& often do you go back & dinero, ¿con que frecuencia \\
another day to win back & vuelve otra vez a jugar para \\
money you have lost? & $\begin{array}{l}\text { recuperar el dinero } \\
\text { perdido? }\end{array}$ \\
\hline
\end{tabular}

Fuente: elaboración propia.

En la versión traducida al español del Test AUDIT, se nota una redundancia léxica en la pregunta 2: “Cuántas bebidas alcohólicas consumes normalmente cuando bebes?". Dado que ya menciona que lo que se está consumiendo son "bebidas", se da por hecho que "beber" es la acción que se realiza.

\subsubsection{Nivel supraoracional}

En este apartado se analizan las cuestiones textuales que no se relacionan con la lengua, es decir, aquellas diferencias en cuanto al contenido. En los cuestionarios estudiados, se detectan diferencias entre las versiones originales y las traducidas. Por ejemplo, en la versión original del cuestionario EAT-26, se observa un formulario en el que se piden los datos del paciente y, en la parte de abajo, otro cuestionario vinculado con el comportamiento, relacionado con el tema del cuestionario (la conducta alimentaria). Por el contrario, en la versión española, el único dato que ofrece el paciente es el nombre en la parte superior del cuestionario y, en lugar de aparecer el segundo cuestionario, se hallan dos páginas en las que se proporciona una breve descripción de este, cómo se interpreta y sus propiedades (la validez y la fiabilidad que describen Martín [2004] y Carvajal et al. [2011], mencionados al inicio de este trabajo).

En el caso del Test AUDIT, se observa que en la versión original del documento se incluyen indicaciones dirigidas al profesional (puesto que está pensado para cumplimentarse en forma de entrevista), que es quien va a leerlo. Sin embargo, estas indicaciones no aparecen en la versión traducida. Otra diferencia está al final del test, 
pues en la versión original se enumeran las instituciones y organizaciones que han creado el documento, el número de personas en las que se ha probado el estudio y los resultados obtenidos, además de una breve descripción del cuestionario y sus propiedades métricas. Por el contrario, en la traducción se ve el Test CAGE, un cuestionario dirigido a personas que ya han sido diagnosticadas con algún problema relacionado con el consumo de alcohol, así como su valoración posterior.

En los demás cuestionarios, no se aprecian diferencias tan evidentes, pero se observan otras, como errores ortotipográficos en las versiones traducidas de los cuestionarios SOGS y BDI-2, tales como inconsistencias en la alineación de las preguntas y las respuestas, dobles espacios o la ausencia de ellos.

\subsection{Cuestiones socioculturales}

En este apartado se analizan las cuestiones vinculadas con la cultura, además de determinar si las versiones están bien adaptadas a los cambios sociodemográficos actuales.

Una de las adaptaciones que debe mencionarse es la del Cuestionario SOGS, en cuyo ítem 2, apartado $f$ ) de la versión original (que se corresponde con el apartado $d$ ) de la versión en español), se observa lo siguiente (tabla 14):

Tabla 14. Ejemplo de adaptación cultural en el cuestionario SOGS

\begin{tabular}{lll}
\hline Cuestionario & Texto original & Texto meta \\
\hline SOGS & f. played the numbers or & d) jugar a la lotería, a \\
& bet on lotteries & quinielas, a la primitiva, a \\
& la bonoloto o a los ciegos.
\end{tabular}

Fuente: elaboración propia.

En el texto meta se produce una adaptación cultural al añadir información relacionada con los juegos de apuestas más conocidos en España. El resto de los cuestionarios estudiados enumeran síntomas de la enfermedad (Escala de Ansiedad de 
Hamilton), sentimientos (BDI-2) o conductas de comportamiento (Test Audit y EAT26) que son independientes a la cultura, ya que las personas actúan de la misma forma en cuanto al trastorno que se evalúa en cada caso, por lo que no se muestran adaptaciones de este tipo al no haber sido necesarias.

\subsection{Cuestiones visuales}

En los cuestionarios objeto de análisis se distinguen dos tipos de formato: el soporte web y el PDF. Según la naturaleza del cuestionario, algunos de ellos están pensados para ser leídos por el profesional sanitario (AUDIT), para ser heteroaplicados (Escala de Ansiedad de Hamilton) o autoadministrados (SOGS, EAT-26 y BDI-2). Se cree que ambos formatos resultan fáciles y cómodos para la cumplimentación de los cuestionarios, aunque el soporte web permite cambiar la respuesta, por ejemplo.

Esta diferencia de formatos afecta el cálculo de resultados, pues en los cuestionarios que están en formato web, se registran de manera automática en las casillas Score, en las que se debe clicar para obtenerlos. Sin embargo, en aquellos documentos en formato PDF, los resultados los calcula el profesional sanitario y los introduce él mismo en los espacios habilitados. A continuación, se muestra en la figura 1 una de las respuestas seleccionadas, como ejemplo de la comodidad que ofrece este tipo de formato. 


\begin{tabular}{|c|c|c|c|c|c|}
\hline & \begin{tabular}{c|c|} 
Not \\
Present
\end{tabular} & Mild & Moderate & Severe & $\begin{array}{l}\text { Very } \\
\text { Severe }\end{array}$ \\
\hline $\begin{array}{l}\text { 1. Anxious Mood } \\
\text { Worries, anticipation of the worst, fearful anticipation, irritability. }\end{array}$ & • & 0 & 0 & 0 & 0 \\
\hline $\begin{array}{l}\text { 2. Tension } \\
\text { Feelings of tension, fatigability, startle response, moved to tears easily, trembling, feelings of restlessness, } \\
\text { inability to relax. }\end{array}$ & 0 & 0 & 0 & 0 & 0 \\
\hline $\begin{array}{l}\text { 3. Fears } \\
\text { Of dark, of strangers, of being left alone, of animals, of traffic, of crowds. }\end{array}$ & 0 & O & 0 & O & 0 \\
\hline $\begin{array}{l}\text { 4. Insomnia } \\
\text { Difficulty in falling asleep, broken sleep, unsatisfying sleep and fatigue on waking, dreams, nightmares, night } \\
\text { terrors. }\end{array}$ & 0 & 0 & 0 & 0 & 0 \\
\hline $\begin{array}{l}\text { 5. Intellectual } \\
\text { Difficulty in concentration, poor memory. }\end{array}$ & 0 & 0 & 0 & 0 & 0 \\
\hline $\begin{array}{l}\text { 6. Depressed Mood } \\
\text { Loss of interest, lack of pleasure in hobbies, depression, early waking, diurnal swing. }\end{array}$ & 0 & 0 & 0 & 0 & 0 \\
\hline $\begin{array}{l}\text { 7. Somatic (muscular) } \\
\text { Pains and aches, twitching, stiffness, myoclonic jerks, grinding of teeth, unsteady voice, increased muscular tone. }\end{array}$ & 0 & 0 & O & 0 & 0 \\
\hline $\begin{array}{l}\text { 8. Somatic (sensory) } \\
\text { Tinnitus, blurring of vision, hot and cold flushes, feelings of weakness, pricking sensation. }\end{array}$ & 0 & 0 & 0 & 0 & 0 \\
\hline $\begin{array}{l}\text { 9. Cardiovascular Symptoms } \\
\text { Tachycardia, palpitations, pain in chest, throbbing of vessels, fainting feelings, missing beat. }\end{array}$ & 0 & 0 & 0 & 0 & 0 \\
\hline $\begin{array}{l}\text { 10. Respiratory Symptoms } \\
\text { Pressure or constriction in chest, choking feelings, sighing, dyspnea. }\end{array}$ & 0 & 0 & ○ & 0 & 0 \\
\hline $\begin{array}{l}\text { 11. Gastrointestinal Symptoms } \\
\text { Difficulty in swallowing, wind abdominal pain, burning sensations, abdominal fullness, nausea, vomiting, } \\
\text { borborygmi, looseness of bowels, loss of weight, constipation. }\end{array}$ & 0 & 0 & 0 & O & 0 \\
\hline $\begin{array}{l}\text { 12. Genitourinary Symptoms } \\
\text { Frequency of micturition, urgency of micturition, amenorrhea, menorrhagia, development of rigidity, premature } \\
\text { ejaculation, loss of libido, impotence. }\end{array}$ & 0 & 0 & 0 & 0 & 0 \\
\hline $\begin{array}{l}\text { 13. Autonomic Symptoms } \\
\text { Dry mouth, flushing, pallor, tendency to sweat, giddiness, tension headache, raising of hair. }\end{array}$ & 0 & O & 0 & 0 & 0 \\
\hline $\begin{array}{l}\text { 14. Behavior at Interview } \\
\text { Fidgeting, restlessness or pacing, tremor of hands, furrowed brow, strained face, sighing or rapid respiration, } \\
\text { facial pallor, swallowing, etc. }\end{array}$ & 0 & 0 & 0 & 0 & 0 \\
\hline
\end{tabular}

Figura 1. Versión original de la Escala de Ansiedad de Hamilton, soporte web.

Fuente: Tomado de https://psychology-tools.com/test/hamilton-anxiety-rating-scale.

El fondo de la mayoría de los documentos es blanco, independientemente del formato en el que se encuentren. El único cuestionario que se diferencia en este aspecto es la versión española del Test AUDIT, cuyo fondo es gris claro. Aunque el color más apropiado para los cuestionarios, según el criterio de este estudio, es el blanco; se cree que el tono gris escogido es también apropiado, puesto que no dificulta la lectura, no distrae (no es un color llamativo) y es neutro, por lo que tampoco daña la vista.

También cabe destacar que ninguno de los cuestionarios analizados consta de imágenes que dificulten la lectura o distraigan la atención de los pacientes, es decir, son austeros en cuanto a ornamentación, tanto complementaria a la información que en ellos aparece como decorativas. Según el criterio de este estudio es un aspecto positivo, puesto que los pacientes centran su atención en cumplimentar los cuestionarios.

Un aspecto relevante es que todos los cuestionarios, tanto las versiones originales como las traducciones, presentan un diseño de página vertical, a excepción de 
la versión original del BDI-2, que está en apaisado. Se considera que, a pesar de la diferencia con otros cuestionarios, esta característica de este documento no dificulta ni la comprensión ni la lectura, ya que las preguntas y las respuestas no aparecen cortadas y mantienen un orden lógico.

En cuanto a la tipografía, se cree que es adecuada en todos los documentos. Aunque el tipo de letra es distinto entre ellos, en todos es austera, sin ornamentaciones y clara. Empero, en lo que respecta al tamaño, se piensa que es pequeño en las respuestas de la versión española del Test AUDIT y en el documento de la versión original del EAT-26. Además, en el Test AUDIT se aprecia el color gris de la fuente, por lo que dificultaría aún más la lectura a personas mayores o con problemas de vista. También se considera un obstáculo en la cumplimentación de estos cuestionarios el tamaño de las casillas de la versión española del Test AUDIT y de las dos versiones, original y traducida, del EAT-26, ya que es demasiado pequeño. Por último, se encuentran varios errores en la alineación, o falta o presencia de espacios a lo largo del documento traducido. Por ejemplo, no están alineados los puntos en los que hay que marcar la respuesta de la pregunta 1. Además, se aprecia la diferencia que existe en el formato de la pregunta entre el texto original y el meta, que es bastante notable, pues en el original hay una tabla para las respuestas, que además se repite en el ítem 16.

Se presentan errores ortotipográficos en el documento del cuestionario BDI-2. Por ejemplo, en el ítem 9 hay doble espacio entre el valor de la respuesta y la respuesta, en las dos primeras opciones de respuesta. Así lo muestran las figuras 2 y 3: 


\section{Suicidal Thoughts or Wishes}

0 . I don't have any thoughts of killing myself.

1. I have thoughts of killing myself, but I would not carry them out.

2. I would like to kill myself.

3. I would kill myself if I had the chance.

Figura 2. Ítem 9 de la versión original del cuestionario BDI-2.

Fuente: Tomado de https://www.ismanet.org/doctoryourspirit/pdfs/Beck-Depression-InventoryBDI.pdf.

\section{Pensamientos o Deseos Suicidas}

0 No tengo ningún pensamiento de matarme.

1 He tenido pensamientos de matarme, pero no lo haría

2 Querría matarme

3 Me mataría si tuviera la oportunidad de hacerlo.

Figura 3. Ítem 9 de la versión traducida del cuestionario BDI-2.

Fuente: Tomado de https://www.ismanet.org/doctoryourspirit/pdfs/Beck-Depression-InventoryBDI.pdf.

\section{Conclusiones}

Los cuestionarios de salud y calidad de vida pretenden mejorar la atención sanitaria, por lo que es importante que estén bien adaptados lingüística y culturalmente. Además, estos instrumentos ofrecen información a la comunidad científica sobre la incidencia de una enfermedad y la gravedad con la que esta afecta a la población, y a los propios pacientes sobre su estado de salud. El principal objetivo de los cuestionarios es ofrecer un diagnóstico adecuado a las necesidades del paciente, por ende, debe comprender la información que en ellos aparece. Los procesos de traducción y adaptación son importantes, ya que a través de ellos se consigue que los usuarios de las versiones originales y traducidas (en este caso al español) entiendan y asimilen la información de la misma manera. Al analizar las versiones traducidas oficiales de los cuestionarios 
escogidos, se determina la validez lingüística, cultural y visual, y la fiabilidad de su aplicación en la actualidad.

Como se ha visto, las cuestiones lingüísticas que se han encontrado se repiten en todos los documentos, en mayor o menor medida:

- Grado de formalidad, en el lenguaje y en el trato al paciente.

- Inclusiones terminológicas y omisiones, a veces necesarias por cuestiones culturales como forma aclaratoria.

- Gramática, errores por influencia del inglés, principalmente, y adaptaciones correctas que se adecúan a las normas de la lengua meta (el español) y aportan naturalidad al texto.

- Ortografía, por desconocimiento de las normas ortográficas de la lengua española o por errores en la puntuación.

- Redundancias, que podrían omitirse, por vicios del lenguaje oral que acaban influyendo en el discurso escrito.

- Traducciones poco afortunadas, por calcos de estructuras, principalmente por la influencia del inglés, que, en ocasiones, dificultan la comprensión del texto.

- Adaptaciones bien hechas, que consiguen naturalidad en la lengua meta del texto.

Por lo tanto, la hipótesis que se planteó al inicio de este trabajo sobre las cuestiones lingüísticas se refuta, ya que se encontraron errores en todos los cuestionarios. 
En los aspectos socioculturales, se observan buenas adaptaciones en general, lo cual contribuye a que el texto se lea de manera natural y fluida, así como facilitar la comprensión. Se confirma lo que se planteó al inicio, pues no se halló ningún elemento en las versiones meta que suponga un obstáculo cultural para los pacientes en la cumplimentación de los cuestionarios.

En los aspectos visuales, se detectaron diferencias entre los formatos y las disposiciones textuales de los documentos, así como diferencias en los encabezados. Como aspecto positivo, se señaló que el tamaño y el tipo de letra escogidos en la mayoría de los cuestionarios es adecuado, puesto que es claro. En la versión española del Test AUDIT, sin embargo, en las respuestas, es pequeño y está en gris que, según lo comentado con respecto al fondo (gris también) podría suponer una dificultad para personas mayores o con problemas de vista. Por el contrario, el fondo del resto de documentos es blanco y, en cuanto a la ornamentación (imágenes, colores, etc.), todos se caracterizan por ser austeros. Además, se observaron errores tipográficos en varios de los documentos traducidos que se han analizado (SOGS y BDI-2). Por consiguiente, no se confirman las hipótesis de este trabajo, pues, a pesar de haber aspectos positivos, hay elementos que podrían suponer una dificultad para los pacientes en varios de los cuestionarios analizados.

Tras la realización de este análisis, se proponen las siguientes indicaciones, con el fin de que sirvan de guía para traductores y redactores de cuestionarios dirigidos a pacientes:

- Se debe primar la redacción en lengua meta. Deben evitarse estructuras sintácticas engorrosas que dificulten la lectura y la comprensión de los cuestionarios, ya que están dirigidos a un público inexperto y podrían afectar de forma negativa los resultados. 
- Se debe mantener el mismo registro, tanto léxico como de trato al paciente. El uso de un lenguaje especializado dificulta la comprensión de los cuestionarios. Por ello, estos deben contener un lenguaje adaptado al público al cual están dirigidos y haber pasado por un proceso de desterminologización, en el caso de que fuese necesario. Por ejemplo, en lugar de utilizar "astenia", preferir términos como "cansancio" o "debilidad".

- Deben evitarse las omisiones de información, excepto en casos en los cuales intervenga la cultura y el traductor considere que pueden malinterpretarse o no entenderse.

- Las inclusiones deben hacerse en los casos en los que sea estrictamente necesario, como adjetivos, verbos, sustantivos, que aporten al texto meta el mismo sentido que tiene el original. Aportar información que no aparezca en el texto original puede cambiar el sentido en el texto meta e influir en los resultados.

- Debe cuidarse la ortografía, ya que la puntuación, la falta de una tilde, etc., podrían alterar el sentido de una palabra u oración y, como consecuencia, del texto.

- Las preguntas deben formularse en positivo, debido a que si aparecen en ellas partículas negativas puede cambiar el sentido de estas y confundir al paciente a la hora de escoger una respuesta y, por lo tanto, el resultado final se vería afectado. 
- Se debe tener presente siempre el contexto cultural del cuestionario. El traductor debe conocer bien ambas culturas, además de las lenguas, para realizar una buena adaptación lingüística y cultural.

- La tipografía escogida ha de ser austera y el tamaño de letra el adecuado, con el fin de facilitar la lectura del cuestionario. A este respecto también hay que tener en cuenta el espacio del que dispone el paciente para responder a las preguntas, pues dependiendo del tipo de respuesta que requiera se necesitará más o menos: marcar con una " $\mathrm{X}$ ", rodear, desarrollar, etc.

- Los formularios no deben contener elementos que dificulten su cumplimentación o distraigan al paciente. Estos tienen que ser visualmente muy simples, de tal forma que el paciente centre su atención en comprender cada una de las preguntas propuestas y rellenar el formulario.

\section{Referencias bibliográficas}

Carvajal, A., Centeno, C., Watson, R., Martínez, M. y Sanz, Á. (2011). ¿Cómo validad un instrumento de medida de la salud? Anales del Sistema Sanitario de Navarra, 34(1), 63-72.

Congost, N. (2010). El lenguaje de las Ciencias de la Salud: Los cuestionarios de salud y calidad de vida y su traducción del inglés al español (Tesis doctoral en Filología Inglesa). Universidad de Alicante, España.

Congost, N. (2016). Aspectos lingüísticos en la traducción de cuestionarios de salud (británicos y estadounidenses); Linguistic challenges in the translation of British and US health questionnaires. The Journal of Spezialised Translation, 26, 116-135. 
Congost, N. (2018). La traducción de la escala de calidad de vida de la OMS: el cuestionario WHOQOL-BREF; Translation of the WHO quality of life scale: the WHOQOL-BREF questionnaire. The Journal of Spezialised Translation, 30, 312334.

Martín, M. C. (2004). Diseño y validación de cuestionarios. Matronas profesión, 5(17), 23-29.

Ministerio de Sanidad, Consumo y Bienestar Social. (2019). Gobierno de España. Recuperado de https://encuestas.msssi.gob.es/limesurvey/index.php/481849

Organización Mundial de la Salud. (2019). Recuperado de http://www.who.int/es/newsroom/fact-sheets/detail/headache-disorders

Palacios, P. (3 de junio de 2003). Cesta punta - el deporte que cautivó América. Euskoguide: Guía de viaje del País Vasco. Recuperado de https://www.euskoguide.com/es/deportes-vascos/cesta-punta-jai-alai/

Real Academia Española. (2009). Diccionario panhispánico de dudas: Nueva gramática de la lengua española. Madrid, España. Recuperado de http://www.rae.es/recursos/diccionarios/dpd

Real Academia Española. (2010). Diccionario panhispánico de dudas: Ortografía de la lengua española. Madrid, España. Recuperado de http://www.rae.es/recursos/diccionarios/dpd

\section{() $\mathbb{A \theta \Theta}$}

Esta obra está bajo una licencia de Creative Commons Reconocimiento-NoComercial-

$\underline{\text { SinObraDerivada } 4.0 \text { Internacional }}$ 